\title{
Bioprinting Complex Cartilaginous Structures with Clinically Compliant Biomaterials
}

\section{Journal Article}

\section{Author(s):}

Kesti, Matti; Eberhardt, Christian; Pagliccia, Guglielmo; Kenkel, David; Grande, Daniel; Boss, Andreas; Zenobi-Wong, Marcy (D)

Publication date:

2015-12-22

Permanent link:

https://doi.org/10.3929/ethz-b-000107475

Rights / license:

In Copyright - Non-Commercial Use Permitted

Originally published in:

Advanced Functional Materials 25(48), https://doi.org/10.1002/adfm.201503423 


\section{Advanced Functional Materials \\ Bioprinting Complex Cartilaginous Structures with Clinically-Compliant Biomaterials --Manuscript Draft--}

\begin{tabular}{|l|}
\hline Manuscript Number: \\
\hline Full Title: \\
\hline Article Type: \\
\hline Section/Category: \\
\hline Keywords: \\
\hline Corresponding Author: \\
\hline $\begin{array}{l}\text { Additional Information: } \\
\text { Question } \\
\begin{array}{l}\text { Please submit a plain text version of your } \\
\text { cover letter here. }\end{array} \\
\text { If you are submitting a revision of your } \\
\text { manuscript, please do not overwrite your } \\
\text { original cover letter. There is an } \\
\text { opportunity for you to provide your } \\
\text { responses to the reviewers later; please } \\
\text { do not add them here. }\end{array}$ \\
\hline
\end{tabular}

not add them here.
adfm.201503423R1

Bioprinting Complex Cartilaginous Structures with Clinically-Compliant Biomaterials

Full Paper

Bioprinting; Cartilage; tissue engineering; polysaccharides; extracellular matrix particles

Marcy Zenobi-Wong, PhD

ETH Zürich

Zürich, SWITZERLAND

\section{Response}

It is with pleasure that I submit the manuscript "Bioprinting Complex Cartilaginous Structures with Clinically-Compliant Biomaterials" for consideration in Advanced Functional Materials. Due to the exponentially growing interest in bioprinting, we feel the diverse readership of the journal would benefit from the major advancements in bioink development outlined in this manuscript. Here we present a versatile bioprinting platform based on FDA-compliant biopolymers, gellan and alginate. This ink can be printed with high fidelity and crosslinks in a cell-compatible manner into high resolution three-dimensional shapes. The addition of micronized tissue particles to our bioink is a simple method for increasing bioactivity of the inks, which up until now have mostly consisted of 1-2 modified polymers. Tissue particles are being used extensively as injectables in both research and clinical settings and can be directly added and extruded due to their size below one hundred microns. The final innovation of this study is the co-extrusion of a pluronics-based support (also FDA-compliant) alongside the bioink to rapidly crosslink the graft and produce overhanging structures. In summary, this bioink has applications in regenerative medicine or for investigating biological processes in a defined, reproducible, and biologically relevant manner. Our technique is extendable to other tissue types by tuning the crosslinking-dependent mechanical properties and by introducing other extracellular matrix tissue particles to mimic the complexity of the intended tissue. We believe this bioink could greatly expand the use of bioprinting in fields which have not yet benefited from additive manufacturing techniques.

It is gratifying to see that Advanced Functional Materials is publishing high impact, highly-cited bioprinting articles and we hope this paper can make an important contribution to this emerging field.
Corresponding Author Secondary Information:

Corresponding Author's Institution:

Corresponding Author's Secondary Institution:

First Author:

Matti Kesti

First Author Secondary Information:

Order of Authors:
Matti Kesti

Christian Eberhardt

Guglielmo Pagliccia

David Kenkel

Daniel Grande 
Marcy Zenobi-Wong, PhD

Order of Authors Secondary Information:

\begin{tabular}{ll} 
Abstract: & $\begin{array}{l}\text { Bioprinting is an emerging technology for the fabrication of patient-specific, } \\
\text { anatomically-complex tissues and organs. A novel bioink for printing cartilage grafts } \\
\text { was developed based on two unmodified FDA-compliant polysaccharides, gellan and } \\
\text { alginate, combined with the clinical product BioCartilage } \AA \text { (cartilage extracellular matrix } \\
\text { particles). Cell-friendly physical gelation of the bioink occurred in the presence of } \\
\text { cations, which were delivered by co-extrusion of a cation-loaded transient support } \\
\text { polymer to stabilize overhanging structures. Rheological properties of the bioink } \\
\text { revealed optimal shear thinning and shear recovery properties for high fidelity } \\
\text { bioprinting. Tensile testing of the bioprinted grafts revealed a strong, ductile material. } \\
\text { As proof of concept, 3D auricular, nasal, meniscal and vertebral disc grafts were } \\
\text { printed based on computer tomography (CT) data or generic 3D models. Grafts after } 8 \\
\text { weeks in vitro were scanned using magnetic resonance imaging (MRI) and histological } \\
\text { evaluation was performed. The bioink containing BioCartilage supported proliferation of } \\
\text { chondrocytes and, in the presence of transforming growth factor beta-3 (TGF- } \beta 3 \text { ), } \\
\text { supported strong deposition of cartilage matrix proteins. A clinically-compliant } \\
\text { bioprinting method is presented which yields patient-specific cartilage grafts with good } \\
\text { mechanical and biological properties. The versatile method can be used with any type } \\
\text { of tissue particles to create tissue-specific and bioactive scaffolds. }\end{array}$ \\
\hline
\end{tabular}


DOI: $10.1002 /((201503423))$

Article type: Full paper

\section{Bioprinting Complex Cartilaginous Structures with Clinically-Compliant Biomaterials}

Matti Kesti, Christian Eberhardt, Guglielmo Pagliccia, David Kenkel, Daniel Grande, Andreas Boss, Marcy Zenobi-Wong*

M. Kesti, G. Pagliccia, Prof. M. Zenobi-Wong

Cartilage Engineering + Regeneration, ETH Zürich, 8097 Zürich, Switzerland

E-mail: marcy.zenobi@hest.ethz.ch

Dr. C. Eberhardt, Dr. D. Kenkel, Dr. A. Boss

Institute of Diagnostic and Interventional Radiology, 8091 Zürich, Switzerland

Prof. D. Grande

Orthopaedic Research Laboratory, The Feinstein Institute for Medical Research, 11030 New York, USA

Keywords: Biomedical Applications, Tissue Engineering, Hydrogels

Bioprinting is an emerging technology for the fabrication of patient-specific, anatomicallycomplex tissues and organs. A novel bioink for printing cartilage grafts was developed based on two unmodified FDA-compliant polysaccharides, gellan and alginate, combined with the clinical product BioCartilage ${ }^{\circledR}$ (cartilage extracellular matrix particles). Cell-friendly physical gelation of the bioink occurred in the presence of cations, which were delivered by coextrusion of a cation-loaded transient support polymer to stabilize overhanging structures. Rheological properties of the bioink revealed optimal shear thinning and shear recovery properties for high fidelity bioprinting. Tensile testing of the bioprinted grafts revealed a strong, ductile material. As proof of concept, 3D auricular, nasal, meniscal and vertebral disc grafts were printed based on computer tomography $(\mathrm{CT})$ data or generic 3D models. Grafts after 8 weeks in vitro were scanned using magnetic resonance imaging (MRI) and histological evaluation was performed. The bioink containing BioCartilage supported proliferation of chondrocytes and, in the presence of transforming growth factor beta-3 (TGF- $\beta 3$ ), supported strong deposition of cartilage matrix proteins. A clinically-compliant bioprinting method is 


\section{WILEY-VCH}

presented which yields patient-specific cartilage grafts with good mechanical and biological properties. The versatile method can be used with any type of tissue particles to create tissuespecific and bioactive scaffolds.

\section{Introduction}

One goal of bioprinting is the fabrication of living tissues and complete organs for use in regenerative medicine. Traditional manufacturing methods such as mold casting produce grafts with relatively low resolution and require a new mold for each new design. Additive manufacturing techniques are compatible with rapid production of patient-specific grafts, allowing precise control over internal and external architecture and customized mechanical properties. These techniques can be used for printing biological materials together with living cells, hence the term 'bioprinting'. 3D bioprinting offers researchers a unique way of depositing cell-laden biocompatible materials, so called bioinks, in high resolution structures with a line thickness on the order of hundreds of microns. Due to the promise of such $\underline{a}$ technology, several commercial bioprinters have entered the market and bioinks are the subject of intense investigation. ${ }^{[1-3]}$ Bioink formulation is often considered one of the most critical aspects of high resolution cellular bioprinting.

Cellular printing requires a bioink with two key properties, namely printability and cytocompatible crosslinking. The identification of printable polymeric systems is mainly done through rheological evaluation of a material's shear thinning behavior and shear recovery. Shear -thinning correlates directly with a bioink's ability to be extruded at low pressure $(<3$ bar), something which ensures high post-printing cell viability. ${ }^{[4]}$ Shear recovery, on the other hand, relates to the ink's resistance to flow after printing, which ensures high fidelity of the printed structure. The presence of cells, however, greatly restricts the crosslinking options as physiologic temperature and $\mathrm{pH}$ need to be maintained and harsh chemicals avoided. Hydrogel bioinks can be crosslinked via covalent or physical interactions or a combination 


\section{WILEY-VCH}

thereof. Ultraviolet light initiated crosslinking of (meth)acrylated polymers has been used most often in bioinks, but the presence of potentially toxic monomers and photoinitiators may complicate clinical translation. ${ }^{[5-8]}$ Physically crosslinked gelation based on temperature, hydrophobic/hydrophilic or ionic interactions has been utilized for pre-crosslinking of several bioink materials including poly(N-isopropylacrylamide) conjugated hyaluronan (HApNIPAAm), ${ }^{[9]}$ gelatin, ${ }^{[10,11]}$ alginate, ${ }^{[12]}$ and gellan. ${ }^{[13]}$ Pre-crosslinking before printing or directly during deposition to stabilize the printed lines is generally followed by a final crosslinking which further increases the mechanical properties and stabilizes the whole structure.

For cartilage engineering applications, natural polymers from animal or plant sources including alginate, collagen, gelatin, gellan and hyaluronan have been all intensively studied as possible bioink materials. ${ }^{[14-17]}$ Limitations associated with the use of single-component hydrogel systems, coupled with the need for a mechanically strong biocompatible material that can withstand the physical demands of the joint, have prompted researchers to consider extracellular matrix (ECM) itself as a scaffold material for tissue engineering. [18-20] Furthermore, the combined effect of ECM particles with TGF- $\beta$ has been shown to significantly increase the chondrogenic potential of primary chondrocytes. ${ }^{[14]}$ Commerciallyavailable allograft cartilage fragments including BioCartilage ${ }^{\circledR}$ (Arthrex) are already used in clinical treatment of articular lesions. BioCartilage is a decellularized and dehydrated off-theshelf product which is used in conjunction with marrow stimulation, -autologous blood, platelet rich plasma (PRP) and fibrin to treat cartilage defects. ${ }^{[21]}$ With a particle size ranging from a few micrometers to hundreds of micrometers, it can be used in extrusion bioinks directly after sieving.

We developed a cartilage-specific bioink for bioprinting applications based on a blend of gellan and alginate (Bioink) which can be pre-crosslinked with cations and mixed with commercially-available BioCartilage particles. All three components of the bioink are already 


\section{WILEY-VCH}

in medical use, ${ }^{[22-25]}$ thus avoiding the extensive regulatory hurdles faced by many other bioinks. The bioink was characterized with BioCartilage (Bioink+BioCartilage) and with hydroxyapatite particles (Bioink+HA) to demonstrate printability independent of particle type. To evaluate printability, three bioink compositions (Bioink, Bioink+BioCartilage and Bioink+HA) were characterized with rheology. Clinically relevant, full-sized grafts were printed using either CT data or generic 3D models created for this study. Printed structures were imaged using MRI to compare the 3D shape with the original model and to evaluate the potential of MRI to detect changes in water relaxation times related to extracellular matrix production in tissue engineered grafts. ${ }^{[26,27]}$ To evaluate cartilage formation, cell-laden Bioink and Bioink+BioCartilage discs were cultured for 8 weeks in vitro with and without TGF- $\beta 3$ supplementation.

\section{Results}

\subsection{Bioink crosslinking}

The bioink described here is a blend of gellan and alginate mixed with human micronized BioCartilage or HA particles $(\leq 40 \mu \mathrm{m}$ size $)$. Gellan is a linear anionic polysaccharide composed of tetrasaccharide repeating units (1,3- $\beta$-D-glucose, 1,4- $\beta$-D-glucuronic acid, 1,4- $\beta$ D-glucose, 1,4- $\alpha$-L-rhamnose). The carboxyl side group on the glucuronic acid is responsible for the gelation behavior of the molecule. Upon cooling, the coiled polymer forms doublehelices (coil-helix transition). Upon addition of mono-, di- or trivalent cations, gelation (solgel transition) occurs as the helices aggregate into junction zones which are linked into a three dimensional network via the coiled part of the molecule. ${ }^{[28-31]}$ This gel formation differs greatly from that of alginate, where the divalent cations bind guluronic acids blocks (Gblocks) and form egg-box structures between chains. ${ }^{[32-34]}$ The gelling behavior of the blended bioink is illustrated using the bioprinted auricular cartilage as an example (Figure 1). The printing process was divided into three stages. To start, the bioink was loaded in the 


\section{WILEY-VCH}

syringe (opaque) and the support polymer into a second syringe (transparent). At this stage, a small amount of cations were present in the bioink to increase viscosity and enhance printing properties (Figure 1a). During co-extrusion of the support, cations diffused to the periphery of the printed auricle where they initiated crosslinking (Figure 1b). After the final structure was completed, the support was eluted in $4^{\circ} \mathrm{C}$ cation-supplemented medium (Figure 1c). The molecular representation of the bioink during crosslinking is illustrated schematically, where the formation of junction zones of gellan and binding of cations within the inter-penetrating hydrogel network around the cells and particles can be seen (Figure 1b-c). Immersing the printed constructs into $4^{\circ} \mathrm{C}$ medium is a cell friendly crosslinking process that has been previously shown to have no effect on chondrocyte viability after printing. ${ }^{[9,12]}$

\subsection{Rheological analysis}

Rheological properties of the Bioink, Bioink $+\mathrm{HA}$, and Bioink+Cartilage Particles were measured to determine the shear behavior and shear recovery, two of the most important predictors of bioink printability. All of the bioink compositions showed shear thinning behavior which is critical for extrusion (Figure 2a). Furthermore, all the compositions had a yield point (weak gel formation) prior to extrusion which is important in preventing particle and cell sedimentation in the syringe (Table 1). The shear recovery curves (Figure 2b) illustrate initial interaction between the measuring probe and the material before the first shear sequence is applied and the polymer chains begin to align. Shear recovery after the second shear sequence was $98 \%$ in Bioink+Cartilage Particles and $90 \%$ in Bioink+HA after ten seconds. At the same time the Bioink alone recovered to only $21 \%$ of the original modulus. Figure 2c illustrates the storage modulus $\mathrm{G}^{\prime}$ after cation-induced crosslinking of Bioink alone where effect of cation concentration and type were investigated. By varying these parameters, properties ranging from a few kilopascals to hundreds of kilopascals could be attained. Based on the maximum $G^{\prime}$ and the ratio between storage and loss moduli $\left(G^{\prime} / G^{\prime \prime}\right)$, an indicator of the 


\section{WILEY-VCH}

elasticity of the material, crosslinking with $20 \mathrm{mM} \mathrm{SrCl}_{2}$ was chosen for the rest of the studies.

Figure $2 \mathrm{~d}$ illustrates the final storage modulus for the three bioink compositions. The Bioink alone had the highest final storage modulus $(152 \mathrm{kPa} \pm 3 \mathrm{kPa})$ compared to Bioink+Cartilage Particles $(96 \mathrm{kPa} \pm 1 \mathrm{kPa})$ and Bioink+HA $(110 \mathrm{kPa} \pm 2 \mathrm{kPa})$, suggesting that crosslinking is somewhat hindered by the particles irrespective of their source.

\subsection{Mechanical properties and swelling behavior}

Mechanical properties of the bioprinted cartilage grafts were assessed in tension. Tensile dumbbell specimens were printed using Bioink+HA particles with or without cells and kept in cell culture medium in a $\mathrm{CO}_{2}$ incubator for 48 hours. HA particles were used to avoid potential confounding interactions between cells and particles. The nozzle path (printing direction) in the gage section of the specimen was chosen to be parallel to the direction of tension (Figure 3a). Young's modulus was significantly higher in acellular constructs (E=230 $\mathrm{kPa} \pm 7.0 \mathrm{kPa})$ compared to cellular ones $(\mathrm{E}=116 \mathrm{kPa} \pm 6.8 \mathrm{kPa})(\mathrm{p}<0.001)$, suggesting that the cells increase the compliance of the construct and/or inhibit the crosslinking. On the other hand, there was no difference in failure strain between the acellular $(37 \% \pm 6.4 \%)$ and cellular $(34 \% \pm 2.1 \%)(\mathrm{p}=0.54)$ constructs

Swelling of the bioink with and without particles was quantified to assess the total water retention and the water retention after gel crosslinking (Figure 3c-d). All calculations were done after the high density particle weight was subtracted from the measured weights to compare the true percentual influence of the particles on swelling. Swelling at $37^{\circ} \mathrm{C}$ up to 48 hours increased the hydrogel weight between $2000-3800 \%$ of the dry weight of the sample which is typical of hydrogels and between $26 \%$ and $54 \%$ of the crosslinking weight of the hydrogels. All the bioink compositions were fully hydrated after 24 hours and more specifically Bioink and Bioink+BioCartilage were fully hydrated after 5 hours suggesting faster swelling kinetics. Comparison between swelling ratios of the Bioink alone and the 


\section{WILEY-VCH}

particle containing compositions after 48 hours suggested dependency on the particle type. The Bioink+HA had statistically significantly lower $(\mathrm{p}<0.001)$ equilibrium swelling ratio than Bioink+BioCartilage and Bioink alone which were similar at 48 hours. These results suggest higher water retention in the presence of the BioCartilage compared to HA particles.

\subsection{Bioprinted structures}

Personalized medicine applications will require high resolution patient-specific data to create high resolution models for bioprinting. As illustration, an auricular cartilage model was created from a CT scan showing the feasibility of 3D printing directly from clinical image data (Figure 4a-c). The printed auricular grafts required co-extrusion of support material due to the overhanging helix up to 116 degrees. The co-extrusion of the support material was shown to preserve horizontal bioink lines without sagging and the printed shape accurately after elution of the support (Figure 4d-f).

Cartilaginous grafts including meniscus (Figure 4g-j), intervertebral discs (Figure 4k) and nose (Figure 4l, m) were printed based on generic models. Cartilage samples were flexible in handling and could be sutured (Figure 4h-j). Two-component intervertebral disc grafts were printed with Bioink+Cartilage particles stained red with food color to localize the nucleus pulposus and with Bioink $+\mathrm{HA}$ to localize peripheral structures corresponding to the annulus fibrosus. All of the structures preserved high shape fidelity and were printed in actual size. The printed structures matched the dimensions of the computer models and were stable in long term culture. For example, nose models were $3.1 \mathrm{~cm}$ in length, $2.6 \mathrm{~cm}$ in width and $1.5 \mathrm{~cm}$ in height to represent a young adult size. ${ }^{[35]}$ Internal nasal cavities were left hollow and represented approximately $31 \%$ of the total volume (Figure $4 \mathrm{l}, \mathrm{m}$ ). These structures were printed without support structure due to small increments in overhanging structures.

\subsection{Bioink biocompatibility}




\section{WILEY-VCH}

Cellular bioprinting process was investigated with Bioink+HA to exclude all the interactions and proliferation cues between particles and cells. One layer thick discs were printed to assess the cell viability after printing (Figure 5a) compared to initial viability of the cells prior to their being mixed into the bioink composite. To investigate cell viability in large structures, a young adult size $\underline{d}$ nose $(3.1 \mathrm{~cm}, 2.6 \mathrm{~cm}$ and $1.5 \mathrm{~cm})$ was printed and kept in static culture until the cell viability in the middle of the construct was evaluated from a central slice (minimum diffusion distance of $5 \mathrm{~mm}$ ). Bioprinting with the particles showed an $80 \%$ viability three hours after printing, however, after four days the cell viability recovered to $97 \%$ where it remained until the end of the experiment. The young adult sized nose graft had decreased viability in the center of the scaffold (60\% viable cells at day 7) compared to $96 \%$ viability in the periphery (Figure 5b). This suggests the need for incorporating internal porosity or channels to enhance nutrition transport. Such nutrition channels ${ }^{[10]}$ or engineered porosity ${ }^{[36]}$ could be incorporated into the bioprinted structures by extruding the support polymer within the grafts, which could later be cleared in subsequent washing/crosslinking steps. With this technique a complex 3D interconnected porous network could be created that could be used to perfuse the grafts with nutrient-rich medium. To further enhance mass transport of nutrients, grafts could also be pre-conditioned in dynamic bioreactors.

The effect of BioCartilage and TGF- $\beta 3$ supplementation on cell proliferation was evaluated in casted gels cultured for 21 days. The Bioink alone did not stimulate cell proliferation; in fact there was a loss in DNA at day 7 which slowly recovered. Bioink+BioCartilage, on the other hand, stimulated proliferation and caused a statistically significant increase $(\mathrm{p}<0.001)$ in DNA over 21 days. With TGF- $\beta 3$ supplementation, there was a statistically significant increase in DNA in the BioCartilage containing samples at day $7(\mathrm{p}<0.001)$. By day 21, both bioinks showed increases in DNA, which were not statistically significantly from each other. Initial amount of DNA was the same for all four groups, showing that BioCartilage itself did not contain significant amounts of DNA residues. 


\section{WILEY-VCH}

\subsection{Extracellular matrix production and cartilage formation}

Cartilage extracellular matrix production was evaluated with histology and immunostaining after 3 (Figure S1) and 8 weeks (Figure 6) in culture. Histological evaluation after 3 weeks revealed a clear increase in cell number, GAG synthesis and collagen II production in both bioink compositions supplemented with TGF- $\beta 3$. Furthermore, Bioink+BioCartilage without growth factors stimulated cell proliferation above Bioink alone which was clearly visible with 3 and 8 week H\&E staining. At both time points the Bioink+BioCartilage showed a slight increase in Alcian blue staining and at the 8 week time point a slight collagen II staining was observed suggesting the need for additional growth factor stimulation. Cells were often seen proliferating around the particles without the growth factor supplementation which suggests that cell-particle adhesion and/or growth factors in the particles are important. However, because in the Bioink+BioCartilage with TGF- $\beta 3$ samples, no site-specific proliferation was observed, the results suggest rather the particles are a source of mitogenic growth factors and not specific cell-matrix adhesive cues. After 8 weeks, the gross appearance of the scaffolds (same order as histology) prior to fixing and paraffin embedding suggested growth factor stimulation had a clear effect on cartilage matrix production as seen in the size and opaque appearance of TGF- $\beta 3$ supplemented samples_(Figure 6). At 8 weeks, both supplemented bioink compositions showed a significant increase in cartilage ECM components and had areas which began to resemble the cell density and GAG content of native cartilage. Furthermore, collagen II deposition was strong throughout the graft in the growth factor supplemented conditions while only pericellular staining was seen in the samples cultured without TGF $\beta$. Collagen type I and alizarin red staining were performed to determine the fibrocartilage production and calcification. Collagen I was found in Bioink+BioCartilage and in both TGF- $\beta 3$ supplemented conditions suggesting some fibrocartilage production, perhaps 


\section{WILEY-VCH}

due to the passaging of the cells. In all the conditions calcification was absent suggesting the cartilage phenotype of the chondrocytes was stable.

\subsection{Magnetic resonance imaging}

To assess the shape retention of the printed structures several MRI techniques were evaluated. The printed nose was kept in PBS for 2 weeks to assure complete swelling prior T2-weighted MR imaging. These images were thresholded and converted into a .STL file (Figure 7c) and compared to the original model used for printing (Figure $7 \mathrm{a}$ ) and to the cartilaginous graft immediately after printing (Figure 7b). Comparison of the original model and the printed graft illustrates precise material extrusion and detailed structures. However, slightly thicker nostril walls were observed in comparison to the original model (white arrows). Furthermore, when comparing the printed structure to the MRI model after 2 weeks swelling, a slight thickening of the nostril walls were observed, however, no sign of degradation or deterioration of the shape was detected.

To investigate cell-seeded graft stability between day 1 and 8 week, Bioink+BioCartilage samples with and without TGF- $\beta 3$ supplementation were measured with MRI. The apparent diffusion coefficient (ADC) and water relaxation times (T1) and (T2) were performed and compared to bovine hyaline cartilage. Diffusion imaging is increasingly applied for noninvasive tissue monitoring and $\mathrm{ADC}$ values provide quantitative information on passive water diffusion restriction and collagen structure. Furthermore, T1 and T2 have been shown to correlate with mechanical properties of native and engineered tissues ${ }^{[26,37,38]}$. T1, T2 and ADC values were acquired from the samples illustrated in Figure 7d-f and there was no significant change in any of these parameters over the culture period suggesting that the graft was stable. All of the values for the engineered tissue samples where higher than for native articular cartilage (Table S1). 


\section{WILEY-VCH}

\section{Discussion}

Bioprinting is a manufacturing method where cell-laden bioinks are deposited with high precision based on computational 3D models. To explore translational possibilities of this technique, clinical sized cartilage structures were bioprinted based on either patient-derived CT or generic 3D models. Previously the external ear shape has been acquired using techniques such as photogrammetry, ${ }^{[39]} \mathrm{MRI},{ }^{[40]}$ and CT, ${ }^{[41]}$ to obtain the three-dimensional models for negative mold fabrication. Nimeskern et al used MRI to manually segment the cartilaginous structure from surrounding tissues. ${ }^{[42]}$ Our bioink printing process combined with one of these imaging techniques could provide a more advanced approach to clinical craniofacial cartilage reconstruction where patient-specific cartilaginous shape is of interest. The present study introduces a newly developed cartilage bioink composite. Both base polymer components of the bioink are already in medical use, thus the clinical translation is likely to be more straightforward than for other bioinks. Gellan has been extensively used in drug delivery, ${ }^{[22]}$ whereas alginate has been clinically used for decades especially in drug delivery and wound healing applications. ${ }^{[23,24]}$ The unique feature of this bioink, however, was the incorporation of cartilage matrix particles to make the printed structures a better biological mimic of native cartilage. Commercial BioCartilage particles have shown potential in treating articular cartilage lesions and were therefore investigated for their effect on proliferation and chondrogenesis in the presence and absence of TGF- $\beta 3$ supplementation. ${ }^{[21,25,43]}$ Other 'micronized' particles can also be incorporated into the bioink formulation to make tissue-specific inks. In this paper, we also demonstrated the excellent printability of HA-containing bioinks, suggesting that tissue particles from any source do not greatly affect the rheological and printing properties of the bioink. Clinically translated and well established decellularized skin, ${ }^{[44,45]}$ and placenta/amnion, ${ }^{[46]}$ have been recently micronized to increase applicability in clinical soft tissue reconstruction as injectables, ${ }^{[47]}$ (Cymetra®, Graftjacket ${ }^{\circledR}$ Xpress, AmnioFix $\left.{ }^{\circledR}\right)$ while clinical particles from bone (DMB ${ }^{\circledR}$ inject ${ }^{\mathrm{TM}}$, Allomatrix $\left.{ }^{\circledR}\right)$ and 


\section{WILEY-VCH}

experimental particles from spinal cord, ${ }^{[48]}$ and small intestinal mucosa ${ }^{[49]}$ amongst others, have been explored primarily as injectables for regenerative medicine, but could easily be translated to bioprinting approaches.

Rheological characterization showed that all bioink compositions underwent shear thinning which is important for bioink extrusion (Figure 2a). During shear thinning, the coiled polymer chains align and disentangle at higher shear rates requiring less extrusion force to deposit the bioink, which is beneficial for cell survival. Billiet et al. recently performed a parametric study investigating the correlation of pressure to cell viability and found increasing pressure ( $p>2$ bar) to have a negative effect on the cell viability. ${ }^{[16]}$ Aguado et al. suggested initial post-printing cell death was due to mechanical cell membrane disruption from high shear stress in the printing nozzle. ${ }^{[4]}$ As shown in Figure 5 the viability in our bioink was $80 \%$ three hours after printing, however, it recovered to $97 \%$ four days after printing and remained high until the end of the experiment. The initial drop in cell viability might be due to partial disruption of the cell membrane as the cells are mixed into the bioink and/or during extrusion itself.

Another important rheological characteristic of bioinks is the fast shear recovery which predicts rapid cessation of flow after extrusion. This property was pronounced in bioinks with particles compared to the Bioink alone. Ten seconds into shear recovery, Bioink+BioCartilage and Bioink+HA had recovered $98 \%$ and $90 \%$ respectively of the original modulus, while Bioink alone had only recovered 21\%. In fact the Bioink required 25 seconds to recover 50\% of the final modulus which is substantially longer time compared to particles containing bioinks. Nearly instantaneous cessation of the particle-containing bioinks can be due to the particles hindering the tight packing of gellan tight junctions and preventing their cationic interactions which lead to faster recovery after shear. Furthermore, after shear the particles induce drag forces acting in opposition to the relative motion of the polymers leading to faster cessation. Several cation concentrations and combinations were investigated based on 


\section{WILEY-VCH}

previous knowledge about cation interactions with the biopolymers. ${ }^{[50,51]}$ Interestingly, barium was found to hinder the crosslinking of pure gellan leading to phase separation with the highly crosslinked alginate. The best crosslinking conditions were achieved with $20 \mathrm{mM}$ strontium chloride solution, where the storage modulus of Bioink $(152 \mathrm{kPa} \pm 3 \mathrm{kPa})$ was higher than Bioink+BioCartilage $(96 \mathrm{kPa} \pm 1 \mathrm{kPa})$ and Bioink $+\mathrm{HA}(110 \mathrm{kPa} \pm 2 \mathrm{kPa})$. Interestingly for cartilage applications, strontium chloride and strontium renalate have been found to induce proteoglycan synthesis in human chondrocytes. ${ }^{[52,53]}$ In fact, Urban et al. suggested that in osteoarthritis, proteoglycan synthesis could decrease due to below physiological ionic strength environment and that additional cation administration could have beneficial effects on chondrocyte metabolism. ${ }^{[54,55]}$ Strontium-based crosslinking of engineered cartilage constructs might therefore have an added benefit of promoting proteoglycan synthesis, thought this remains to be tested.

One concern with bioprinted structures are structural micro-defects which effect material properties of the crosslinked grafts. ${ }^{[56]}$ These defects include layer-layer and thread-thread adhesion depending on the crosslinking kinetics and in this case crack nucleation sites due to the presence of the particles and cells. We found the tensile modulus of Bioink+HA decreased when cells were added to the ink (from $230 \mathrm{kPa} \pm 7.0 \mathrm{kPa}$ to $116 \mathrm{kPa} \pm 6.8 \mathrm{kPa}$ ), but there was no difference in failure strain between the acellular $(37 \% \pm 6.4 \%)$ and cellular $(34 \% \pm$ $2.1 \%)$ constructs. The difference in tensile modulus was significant and might be partly due to the tensile measurement itself, where each of the constituent components effects the final modulus. ${ }^{[56]}$ In this case, the cells increase the volume fraction of the soft components and might hinder the network formation. Furthermore, the stress-strain curve with rigid HA particles has a clear yield point whereas the cell-containing material has a prolonged yield area and a more linear stress-strain response. In comparison to the tensile modulus of native superficial zone articular cartilage $(E=4.98 \pm 1.66 \mathrm{MPa}){ }^{[57]}$ the tensile modulus of the printed grafts is an order of magnitude lower; however, the linear regions of the stress-strain 


\section{WILEY-VCH}

curves imply native cartilage-like elasticity, ${ }^{[58]}$ where cyclic loading within the $10-15 \%$ of strain would fully recover. Mechanical test data suggest that while the particle embedded grafts are still mechanically inferior compared to the native cartilage, it may be appropriate as a temporary structure for tissue formation especially in non-weight bearing craniofacial applications. The mechanical properties of the printed structures could be enhanced by several means including longer crosslinking times, the use of reinforcement scaffolds and longer pre-culture to allow newly synthesized ECM proteins to be deposited within the structure.

To investigate water retention after crosslinking and its effect on shape retention, swelling studies and MR imaging were performed. The water retention of the bioink with and without particles was investigated and some particle-specific interactions were observed. Bioink had a water uptake of $3000 \%$ compared to a dry polymer weight which corresponds to literature values for low acylated gellan gels. ${ }^{[59]}$ The total water retention after 48 hours was significantly different in Bioink+HA (2000\%) and Bioink+BioCartilage (3800\%) compared to the Bioink alone. Similarly, the equilibrium swelling after the hydrogel crosslinking illustrated similar behavior where the weight of the Bioink+HA, Bioink+BioCartilage and Bioink increased 26\%, 52\%, and 54\% respectively. Increased water retention was observed in the presence of BioCartilage particles which contain negatively charged GAGs known to attract water molecules whereas HA particles are electrostatically neutral and decreased the overall sample swelling due to decreased volume fraction of the bioink. Furthermore, MRI was performed to assess shape and size changes of the nose grafts. When the original model was compared to the printed cartilaginous graft, slight swelling was observed (Figure 7). Methods to overcome this would be to reduce the geometry of the graft in anticipation of the swelling process or to increase the crosslinking density of the grafts.

Cell-laden Bioink and Bioink+BioCartilage were cultured for 8 weeks in vitro to evaluate their potential for supporting chondrocyte proliferation and cartilage formation. Histologic 


\section{WILEY-VCH}

evaluation of the tissue engineered samples (Figure 6) illustrates cartilage matrix production where the gross appearance was clearly different in both TGF- $\beta 3$ supplemented groups. The increase in size and opaqueness suggests ECM synthesis and formation of cartilaginous matrix. Further histological evaluation revealed cell proliferation in Bioink+BioCartilage and to a higher extent in both TGF- $\beta 3$ supplemented groups. MRI measurements performed prior to the histologic evaluation of the same samples however showed that while T1, T2 and ADC values could detect differences between native and engineered cartilage, they could not detect changes in matrix deposition over the 8 week culture period. Recently, Chuck et al. performed similar studies using MRI to monitor changes during muscle formation in vivo. The injected stem cells formed cell clusters that decreased in T1, T2 and ADC nearly to the levels of native muscle tissue in 28 days. ${ }^{[26]}$ This difference in MRI sensitivity might be related to the inherently higher water content of the bioink (Figure 3c, d) due to its softer and extensively hydrated polymeric network compared to the dense cellular structures. Furthermore, the dense highly crosslinked network of type II collagen and GAGs seen in articular cartilage, leading to lower water diffusion and relaxation times, may have not yet developed in the engineered grafts.

\section{Conclusions}

In summary, high resolution and viable cartilage grafts were successfully bioprinted with the bioink composite. We show with this bioink that extracellular matrix particles can be reconstituted into de novo bioprinted cartilaginous structures. This technique is extendable to all sorts of tissue particles and their combinations and is a promising, simple and clinically compatible approach to extend the bioactivity of bioinks to towards that of native tissues. The material properties of the structures can be further tuned by the chosen cations, concentration and duration of the ionic crosslinking. 


\section{WILEY-VCH}

\section{Experimental Section}

Materials: Gellan (Gelrite) was purchased from the Sigma-Aldrich (Buchs, Switzerland) and further purified to remove residual cations. Ultrapure, high $\mathrm{G}$ content alginate (ProNova UP-LVG) was purchased from NovaMatrix (Sandvika, Norway). Pluronic F127 and D-glucose was purchased from Sigma-Aldrich (Buchs, Switzerland). Dulbecco's modified Eagle's media (DMEM), phosphate buffered saline (PBS), fetal bovine serum (FBS), penicillin-streptomycin (PS), and trypsin were all purchased from Life Technologies (Zug, Switzerland). Dialysis membranes were purchased from SpectrumLabs (Breda, Netherlands). All concentrations are given in percentages weight/volume (\% w/v) unless indicated otherwise.

Bioink preparation: Gellan was purified from residual cations by dialyzing against ethylenediaminetetraacetic acid (EDTA) $(0.001 \%)$ in ultra-pure water to decrease the temperature of solubility and sol-gel transition. Purified gellan was added to D-glucose $(300 \mathrm{mM})$ in ultra-pure water at $90^{\circ} \mathrm{C}$ to achieve a $6 \%$ solution. The boiling flask was kept at $90^{\circ} \mathrm{C}$ with agitation until the solution was homogeneous. $4 \%$ alginate solution was dissolved in D-glucose supplemented ultra-pure water. A 50:50 ratio of both polymer solutions were mixed to obtain a $3 \%$ gellan and $2 \%$ alginate blend. The temperature was reduced to $38-40^{\circ} \mathrm{C}$ before adding the particles of choice in $40 \% \mathrm{w} / \mathrm{w}$ (particle/ total polymer) concentration. When printed with cells $\left(6 \times 10^{6}\right.$ cells $\left./ \mathrm{ml}\right)$ or without cells, DMEM solution was added to the solution in 1:10 volume ratio to pre-crosslink the bioink. Mixing was continued until the solution reached room temperature and the printing syringes were loaded. Support material was prepared by dissolving $30 \%$ pluronic in sodium chloride $(150 \mathrm{mM})$ and strontium chloride $(20 \mathrm{mM})$ solution at $4{ }^{\circ} \mathrm{C}$. Support material was loaded into the printing syringes and brought to room temperature prior use. 


\section{WILEY-VCH}

Printing syringes of both the bioink and the support polymer were mounted onto the extrusion printer Biofactory ${ }^{\circledR}($ RegenHu, Switzerland) and the parameters were set for a 410 micron nozzle diameter. Two pneumatic extrusion heads with adjustable extrusion pressure up to 6 bar were used in parallel. A pressure of 0.2 bar and flow rate of $800 \mathrm{~mm} / \mathrm{min}$ were used with Bioink and Bioink+HA. Bioink+BioCartilage required increased pressure of 1 bar for precise extrusion at the same printing speed. Support ink was co-extruded using 1.5 bar pressure and $800 \mathrm{~mm} / \mathrm{min}$ flow rate. All 3D designs from clinical CT scans (kind gift of Phonak) and other 3D models were made with Cinema 4D software (Maxon, Germany) and converted into .STL files for the bioprinter. Extrusion printing was initiated with support polymer layer followed by bioink to initiate crosslinking immediately upon contact between the two materials. Immediately following the printing, the grafts were transferred into sterile petri dishes containing $20 \mathrm{mM} \mathrm{SrCl} 2$ supplemented DMEM at $4^{\circ} \mathrm{C}$ and crosslinked for $15-30$ minutes. Following the crosslinking, specimens were washed twice in DMEM containing $10 \%$ fetal bovine serum, $1 \%$ penicillin/streptomycin and L-ascorbic acid $\left(50 \mu \mathrm{g} \mathrm{ml}^{-1}\right)$ and then cultured under normoxic $\left(21 \% \mathrm{O}_{2}\right)$ conditions until analysis.

Rheology: All liquid state measurements were performed in rotation with a plate-plate geometry (20 mm diameter, MCR 301, Anton Paar, Zofingen, Switzerland) saturating the measuring chamber with water vapor to prevent drying. Shear thinning experiments were performed by measuring viscosity $\eta$ at a frequency of $1 \mathrm{rad} \mathrm{s}^{-1}$ with logarithmic increase of shear rate. Yield points were calculated using the Herschel/Bulkley equation

$\tau=\tau_{H B}+c \cdot \dot{\gamma}^{p}$

where $\tau$ is shear rate, $\tau_{\mathrm{HB}}$ is the Herschel/Bulkley yield point, c flow coefficient, $\dot{\gamma}^{\mathrm{p}}$ shear stress with exponent $\mathrm{p}$, where $\mathrm{p}$ is the Herschel/Bulkley index $(\mathrm{p}<1$ for shear thinning and $\mathrm{p}$ $>1$ for shear thickening). 
Shear recovery, also known as structural recovery, was performed by measuring storage modulus $\mathrm{G}^{\prime}$ and loss modulus $\mathrm{G}^{\prime \prime}$ at a frequency of $1 \mathrm{rad} / \mathrm{s}$ and $1 \%$ strain which was determined to be within the linear viscoelastic (LVE) range, then shearing the sample for 1 second at $100 \mathrm{~s}^{-1}$ simulating the printing induced shear before returning to the oscillatory measurement. This shear cycle was repeated twice. Gel state crosslinking experiments were measured in oscillation with plate-plate geometry $(10 \mathrm{~mm}$ diameter) for cation determination to ensure cation diffusion to the core and with (20 $\mathrm{mm}$ diameter) for final gel storage modulus $\mathrm{G}^{\prime}$ to minimize sample slipping. The samples were crosslinked in corresponding cation solutions for 30 minutes before the measurements were recorded. Since the amount of human BioCartilage was limited, rheology experiments were performed using cryomilled and lyophilized cartilage particles $(\leq 40 \mu \mathrm{m}$ in diameter) harvested from calves femoral condyles (Bioink+Cartilage Particles). All the rheological measurements were measured in triplicates except the final storage modulus (Figure 2d) where samples were measured in duplicates.

Mechanical testing: Tension testing was performed (TA.XTplus, Stable Micro Systems, UK) using printed dumbbell-shaped specimens and Bioink+HA to avoid cell and particle interactions during the 48 hour swelling period at $37^{\circ} \mathrm{C}$ in medium. Tensile specimens were $0.9 \mathrm{~mm}$ in thickness comprising two printed layers. Samples were subjected to a controlled tensile displacement of $0.03 \mathrm{~mm} \mathrm{~s}^{-1}$ until failure. Young's modulus was calculated from the linear region of the stress-strain curve, which was until 10\% strain for all the tension samples.

Swelling: For the swelling experiments, bioink gels with and without particles were casted in $40 \mu \mathrm{l}$ discs $(\mathrm{n}=5)$. Gels were weighted for initial casting weight (mCasting) after 15 minutes in crosslinking solution. Gels were then immersed in $1 \mathrm{ml}$ of PBS at $37^{\circ} \mathrm{C}$ and incubated for $0.5,1,4,24$ and 48 hours. After all incubation periods, PBS was removed and the gels were weighed $\left(\mathrm{m}_{\text {Swollen }}\right)$. The gels were then snap-frozen and lyophilized before dry weighting $\left(\mathrm{m}_{\text {Dry }}\right)$. The swelling ratio $\mathrm{Q}$ was then calculated as: 
$\mathrm{Q}=\frac{\mathrm{m}_{\text {Swollen }}-\mathrm{m}_{\text {Dry }}}{\mathrm{m}_{\text {Dry }}}$

where Q represents total water retention in the gels.

To calculate the hydrogel swelling ratio after gel crosslinking, equilibrium swelling was calculated as:

$\mathrm{Q}_{E}=\frac{\mathrm{m}_{\text {Swollen }}-\mathrm{m}_{\text {Casting }}}{\mathrm{m}_{\text {Casting }}}$

where the $\mathrm{Q}_{\mathrm{E}}$ represents the additional water retention after the hydrogels were casted.

All the calculations were done after the high density particle weight was subtracted from the measured weights to compare the true percentual influence of the particles on swelling. Particle weight of $1.6 \mathrm{mg}$ was reduced from both particle containing compositions due to the $40 \mu \mathrm{l}$ gel volume and $40 \% \mathrm{w} / \mathrm{w}$ particle content.

MRI scanning and imaging protocol: MR imaging was performed in a 7.0 Tesla Biospec MR Scanner (Bruker, Ettlingen, Germany) with gradient amplitude of $200 \mathrm{mT} \mathrm{m}^{-1}$ and a maximal slew rate of $640 \mathrm{~T} \mathrm{~m}^{-1} \mathrm{~s}^{-1}$ using a linear polarized mouse whole-body $1 \mathrm{H}$ transmit-receive mouse coil (1H 075/040 QSN, Bruker). All measurements were performed at room temperature $\left(20.5-20.6^{\circ} \mathrm{C}\right)$. After positioning the sample, the coil was adjusted by manual wobbling. After a gradient-echo (GRE) localizer in 3 spatial directions, the morphological integrity was assessed by a T2w Rapid Acquisition with Relaxation Enhancement (RARE) sequence (TR/TE $1800 \mathrm{~ms} / 42 \mathrm{~ms}$; echo train length 16; matrix $128 \mathrm{x}$ 256; FoV 17.5 x 35; slice thickness $0.273 \mathrm{~mm}$, averages 1). Material properties were further characterized by the T1- and T2- relaxation times and water diffusion. T1-longitudinal and T2-transverse relaxation times were evaluated by a fast spin-echo sequence with varying echo-time and repetition time (6 different TRs: 200, 400, 800, 1500, 3000 and $5500 \mathrm{~ms}$ and 


\section{WILEY-VCH}

five different TEs: 8, 25, 42, 59, 76 ms, echo train length 2; matrix $192 \times 256$; FoV 24 x 24

$\mathrm{mm}$; slice thickness $1.0 \mathrm{~mm}$, averages 1), whereas diffusion properties were determined by a diffusion-weighted spin-echo sequence with 4 different $b$-values $\left(b=0,150,350,470 \mathrm{~s} \mathrm{~mm}^{-2}\right.$, TR/TE $2500 \mathrm{~ms} / 22 \mathrm{~ms}$; echo train length 1; matrix 128 x 128; FoV 24 x 24 mm; slice thickness $1.0 \mathrm{~mm}$, averages 1$)$.

MRI data analysis: Custom made in-house post-processing-routines using the programming language Matlab (The Mathworks, Inc, Natick, MA, USA) were used for data analysis. Polygonal RoIs were drawn in triplicate for each ex-vivo cartilage sample in order to assess representatively the respective tissue. The T1-, T2-relaxation times and ADC values were inferred by region of interest (RoI) analysis. In brief, the $\mathrm{T} 1$ and $\mathrm{T} 2$ relaxation times could be retrieved by means of monoexponential fitting of the signal intensities over the repetition times for $\mathrm{T} 1$ determination, respectively the echo times for $\mathrm{T} 2$ determination using a Levenberg-Marquardt non-linear least-squares algorithm. ADC was calculated by a monoexponential fitting of the signal intensities against at the four $b$ values. Mean values and standard deviations were calculated from the computed T1, T2, and ADC values.

Cell isolation and in vitro culture: Bovine chondrocytes were harvested from full thickness articular cartilage of the lateral and medial femoral condyles of four $\sim 6$ month old calves obtained from the slaughter house. Cartilage slices were minced and digested for $6 \mathrm{~h}$ with $0.03 \%$ collagenase in DMEM supplemented with $1 \%$ PS under gentle stirring. The digest was then filtered through a $100 \mu \mathrm{m}$ cell strainer and subsequently through a $40 \mu \mathrm{m}$ cell strainer before the cells were pooled. Passage 0 cells were seeded at $10^{\prime} 000 \mathrm{cells} / \mathrm{cm}^{2}$ in DMEM containing $10 \%$ fetal bovine serum, $50 \mu \mathrm{g} / \mathrm{mL}$ L-ascorbic acid and $1 \%$ PS. This media formulation was used in all further experiments. At $~ 80 \%$ confluency, cells were trypsinized and washed several times with culture media and added to the bioink. Bioink solutions with and without particles were prepared and mixed with passage one (P1) chondrocytes at a density of $6 \times 10^{6}$ cells $/ \mathrm{ml}$. Gels of $40 \mu 1$ in volume were crosslinked for 15 minutes with 


\section{WILEY-VCH}

$20 \mathrm{mM} \mathrm{SrCl} 2$ in DMEM. Cultures with and without supplementation of $10 \mathrm{ng} / \mathrm{ml}$ TGF- $\beta 3$ were carried out for 8 weeks in normoxic $\left(21 \% \mathrm{O}_{2}\right)$ conditions.

Cell viability and proliferation assessment: Cell viability after printing was imaged using $2 \mu \mathrm{M}$ calcein $\mathrm{AM}$ and $10 \mu \mathrm{M}$ propidium iodide staining solution in PBS (Zeiss Axio Observer, Zeiss, Switzerland). Total amount of DNA was assessed with a Picogreen® (Life technologies, Zug, Switzerland) kit.

Histology and immunohistochemistry: Hematoxylin and eosin (H\&E) and Alcian blue stainings were performed after 3 and 8 weeks. Immunohistochemical staining of collagen type $\underline{\text { I and II wereas performed using primary antibody (Abcam, ab34710) and (Rockland, }}$ BioConcept, 600-401-104S) respectively. The secondary antibody labeled with Alexa fluor 488 (LifeTechnologies, Zug, Switzerland) was used and the samples were counterstained with Hoechst. Samples after 8 weeks in vitro were stained with alizarin red and compared to calcified bone samples. Samples were imaged (Zeiss Axio Observer, Zeiss, Switzerland) and evaluated with Image J software.

Statistical analysis: Data from the assays is expressed as mean \pm standard deviation. Statistical analysis of the data was performed using a one-way ANOVA and Bonferroni posthoc testing (OriginPro 8G, OriginLab) and the level of significance was determined at $\mathrm{p}<0.05$.

\section{Supporting Information}

Supporting Information is available from the Wiley Online Library or from the author.

\section{Acknowledgements}

The work was funded by the Swiss National Science Foundation (grant number CR32I3_146338 / 1) and Fifa/F-MARC. Authors would like to thank Geoff Warnock and 


\section{WILEY-VCH}

Mathias Wyss for their MRI expertise and Phonak for the CT ear scans used in this study. The

type II collagen monoclonal antibody developed by T.F. Linsenmayer was obtained from the

Developmental Studies Hybridoma Bank, created by the NICHD of the NIH and maintained at The University of Iowa, Department of Biology, Iowa City, IA 52242.

Received: ((will be filled in by the editorial staff))

Revised: ((will be filled in by the editorial staff)) Published online: ((will be filled in by the editorial staff))

[1] J. Malda, J. Visser, F. P. Melchels, T. Jüngst, W. E. Hennink, W. J. a Dhert, J. Groll, and D. W. Hutmacher, Adv. Mater. 2013, 25.

[2] S. V Murphy, A. Skardal, and A. Atala, J. Biomed. Mater. Res. A. 2013, 101.

[3] D. Seliktar, Science 2012, 336.

[4] B. A. Aguado, W. Mulyasasmita, J. Su, D. Ph, K. J. Lampe, and S. C. Heilshorn, Tissue Eng. Part A 2012, 18, 7 .

[5] W. Schuurman, P. a Levett, M. W. Pot, P. R. van Weeren, W. J. a Dhert, D. W. Hutmacher, F. P. W. Melchels, T. J. Klein, and J. Malda, Macromol. Biosci. 2013, 13.

[6] P. a. Levett, F. P. W. Melchels, K. Schrobback, D. W. Hutmacher, J. Malda, and T. J. Klein, Acta Biomater. 2014, 10, 1.

[7] L. a Hockaday, K. H. Kang, N. W. Colangelo, P. Y. C. Cheung, B. Duan, E. Malone, J. Wu, L. N. Girardi, L. J. Bonassar, H. Lipson, C. C. Chu, and J. T. Butcher, Biofabrication 2012, 4, 3.

[8] A. Skardal, J. Zhang, L. McCoard, X. Xu, S. Oottamasathien, and G. D. Prestwich, Tissue Eng. Part A. 2010, 16, 8.

[9] M. Kesti, M. Müller, J. Becher, M. Schnabelrauch, M. D’Este, D. Eglin, and M. Zenobi-Wong, Acta Biomater. 2015, 11.

[10] S. Wüst, M. E. Godla, R. Müller, and S. Hofmann, Acta Biomater. 2014, 10, 2.

[11] T. Billiet, B. Van Gasse, E. Gevaert, M. Cornelissen, J. C. Martins, and P. Dubruel, Macromol. Biosci. 2013, 13, 11.

[12] J. H. Y. Chung, S. Naficy, Z. Yue, R. Kapsa, A. Quigley, S. E. Moulton, and G. G. Wallace, Biomater. Sci. 2013, $1,7$.

[13] J. T. Oliveira, L. Martins, R. Picciochi, I. B. Malafaya, R. a Sousa, N. M. Neves, J. F. Mano, and R. L. Reis, J. Biomed. Mater. Res. Part A 2010, 93A, 3.

[14] C. R. Rowland, D. P. Lennon, A. I. Caplan, and F. Guilak, Biomaterials 2013, 34, 23. 


\section{WILEY-VCH}

[15] M. Ahearne and D. J. Kelly, Biomed. Mater. 2013, 8, 3.

[16] T. Billiet, E.Gevaert, T. De Schryver, M. Cornelissen, and P. Dubruel, Biomaterials 2014, 35, 1

[17] R. A. A. Muzzarelli, F. Greco, A. Busilacchi, V. Sollazzo, and A. Gigante, Carbohydr. Polym. 2012, 89, 3 .

[18] S. F. Badylak, D. O. Freytes, and T. W. Gilbert, Acta Biomater. 2009, 5, 1.

[19] F. Z. Volpato, T. Führmann, C. Migliaresi, D. W. Hutmacher, and P. D. Dalton, Biomaterials 2013, 34, 21.

[20] F. Pati, J. Jang, D.-H. Ha, S. Won Kim, J.-W. Rhie, J.-H. Shim, D.-H. Kim, and D.-W. Cho, Nat. Commun. 2014, 5.

[21] G. D. Abrams, N. A. Mall, L. A. Fortier, B. L. Roller, and B. J. Cole, Oper. Tech. Sports Med. 2013, $21,2$.

[22] T. Osmałek, A. Froelich, and S. Tasarek, Int. J. Pharm. 2014, 466.

[23] Y. K. Lee and D. J. Mooney, Prog Polym Sci. 2013, 37, 1.

[24] J. Sun and H. Tan, Materials. 2013, 6, 4.

[25] S. Desai, J. foot ankle Surg. 2014, 14.

[26] N. C. Chuck, F. A. Zouraq, M. Rottmar, D. Eberli, and A. Boss, Radiology 2015, 274, 3.

[27] M. Kotecha, D. Klatt, and R. L. Magin, Tissue Eng. Part B 2013, 19, 6.

[28] H. Grasdalen and O. Smidsrød, Carbohydr. Polym. 1987, 7, 5.

[29] V. Crescenzi, M. Dentini, T. Coviello, and R. Rizzo, Carbohydr. Res. 1986, 149, 2.

[30] V. Crescenzi and M. Dentini, Carbohydr. Res. 1987, 160.

[31] M. Dentini, T. Coviello, W. Burchard, and V. Crescenzi, Macromolecules 1988, 21, 11.

[32] A. Haug and O. Smidsrød, Acta Chem. Scand 1962, 16.

[33] A. Haug, B. Larsen, and O. Smidsrød, Acta Chem. Scand 1966, $20,1$.

[34] A. Haug and O. Smidsrød, Acta Chem. Scand 1970, 24.

[35] I.-S. Kim, M.-Y. Lee, K.-I. Lee, H.-Y. Kim, and Y.-J. Chung, Clin. Exp. Otorhinolaryngol. 2008, 1,1 .

[36] M. Müller, J. Becher, M. Schnabelrauch, M. Zenobi-Wong, J. Vis. Exp. 2013, 77.

[37] R. Grenier, D. Perie, G. Gilbert, G. Beaudoin and D. Curnier, J. Biomed. Sci. and Eng. 2014,7.

[38] M. J. Nissi, J. Rieppo, J. Töyräs, M. S. Laasanen, I. Kiviranta, M. T. Nieminen and J. S. Jurvelin, Osteoarthritis and Cartilage 2007, 15, 10. 


\section{WILEY-VCH}

[39] A. J. Reiffel, C. Kafka, K. A. Hernandez, S. Popa, J. L. Perez, S. Zhou, S. Pramanik, B. N. Brown, W. S. Ryu, L. J. Bonassar, and J. A. Spector, PLoS One 2013, 8, 2.

[40] L. Nimeskern, H. Martínez Ávila, J. Sundberg, P. Gatenholm, R. Müller, and K. S. Stok, J. Mech. Behav. Biomed. Mater. 2013, 22.

[41] Y. Liu, L. Zhang, G. Zhou, Q. Li, W. Liu, Z. Yu, X. Luo, T. Jiang, W. Zhang, and Y. Cao, Biomaterials 2010, 31, 8.

[42] L. Nimeskern, E.-M. Feldmann, W. Kuo, S. Schwarz, E. Goldberg-Bockhorn, S. Dürr, R. Müller, N. Rotter, and K. S. Stok, PLoS One 2014, 9, 8.

[43] W. Cole, Surg. Pod. 2013.

[44] L. A. Jansen, P. De Caigny, N. Guay, W. Lineaweaver, and K. Shokrollahi, Ann. Plast. Surg. 2013, 70,5 .

[45] A. Reyzelman, R. T. Crews, J. C. Moore, L. Moore, J. S. Mukker, S. Offutt, A. Tallis, W. B. Turner, D. Vayser, C. Winters, and D. G. Armstrong, Int. Wound J. 2009, 6, 3.

[46] M. Litwiniuk and T. Grzela, Wound Repair Regen. 2014, 22, 4.

[47] G. Yoo and J. S. Lim, J. Korean Med. Sci. 2009, 24, 1.

[48] E. M. Carpenter, C. Llamas, B. E. Buck, and T. I. Malinin, J. Neurosci. Res. 2015, 93, 3.

[49] Y. Zhou, Z. Yan, H. Zhang, W. Lu, S. Liu, X. Huang, H. Luo, and Y. Jin, Tissue Eng. Part A 2011, 17, 23.

[50] E. Miyoshi, T. Takaya, and N. Katsuyoshi, Carbohydr. Polym. 1996, 30.

[51] Y. A. Mørch, I. Donati, B. L. Strand, and G. Skjak-Braek, Biomacromolecules 2006, 7.

[52] Y. Henrotin, A. Labasse, S. X. Zheng, P. Galais, Y. Tsouderos, J. M. Crielaard, and J. Y. Reginster, J. bone Miner. Res. 2001, 16, 2.

[53] J.-Y. Reginster, J. Badurski, N. Bellamy, W. Bensen, R. Chapurlat, X. Chevalier, C. Christiansen, H. Genant, F. Navarro, E. Nasonov, P. N. Sambrook, T. D. Spector, and C. Cooper, Ann. Rheum. Dis. 2013, 72.

[54] J. P. Urban and M. T. Bayliss, Biochim. Biophys. Acta 1989, 992, 1.

[55] J. P. G. Urban, A. C. Hall, and K. A. Gehl, J. Cell. Physiol.1993, 154.

[56] C. T. McKee, J. A. Last, P. Russell, and C. J. Murphy, Tissue Eng. Part B 2011, 17, 3.

[57] S. Akizuki, V. C. Mow, F. Müller, J. C. Pita, D. S. Howell, and D. H. Manicourt, J. Orthop. Res. 1986, 4, 4.

[58] M. Charlebois, M. D. McKee, and M. D. Buschmann, J. Biomech. Eng. 2004, 126, 2.

[59] D. R. Pereira, J. Silva-Correia, S. G. Caridade, J. T. Oliveira, R. A. Sousa, A. J. Salgado, J. M. Oliveira, J. F. Mano, N. Sousa, and R. L. Reis, Tissue Eng. Part C 2011, 17, 10. 
WILEY-VCH

1

2

3

4

5

6

9

10

11

12

13

14

15

16

17

18

19

20

21

22

23

24

25

26

27

28

29

30

31

32

33

34

35

36

37

38

39

40

41

42

43

44

45

46

47

48

49

50

51

52

53

54

55

56

57

58

59

60

61

62

63

64

65 


\section{WILEY-VCH}

a)

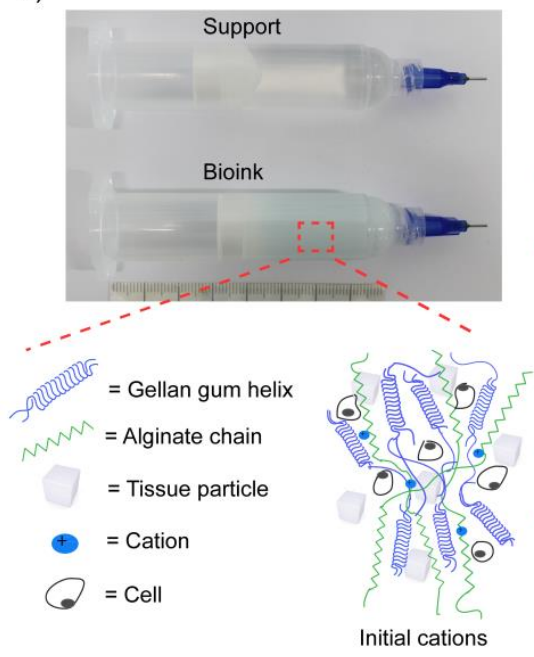

b)

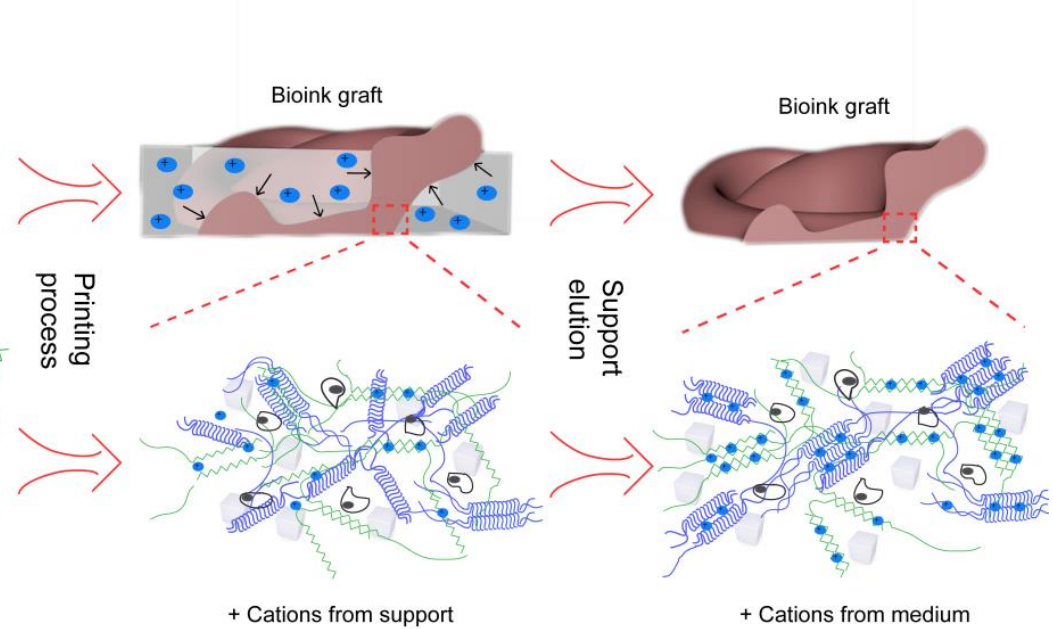

Figure 1. Schematic of the macroscopic and molecular crosslinking process. Support material (transparent) and the bioink (opaque) were loaded into the printing cartridges a). During and after printing the cations diffuse from the support to the periphery of the bioink graft initiating the crosslinking b). After $4^{\circ} \mathrm{C}$ elution and final crosslinking, the graft is self-supporting c). On a molecular level, the gellan helices and alginate chains are increasingly crosslinked at each stage of the process. 


\section{WILEY-VCH}
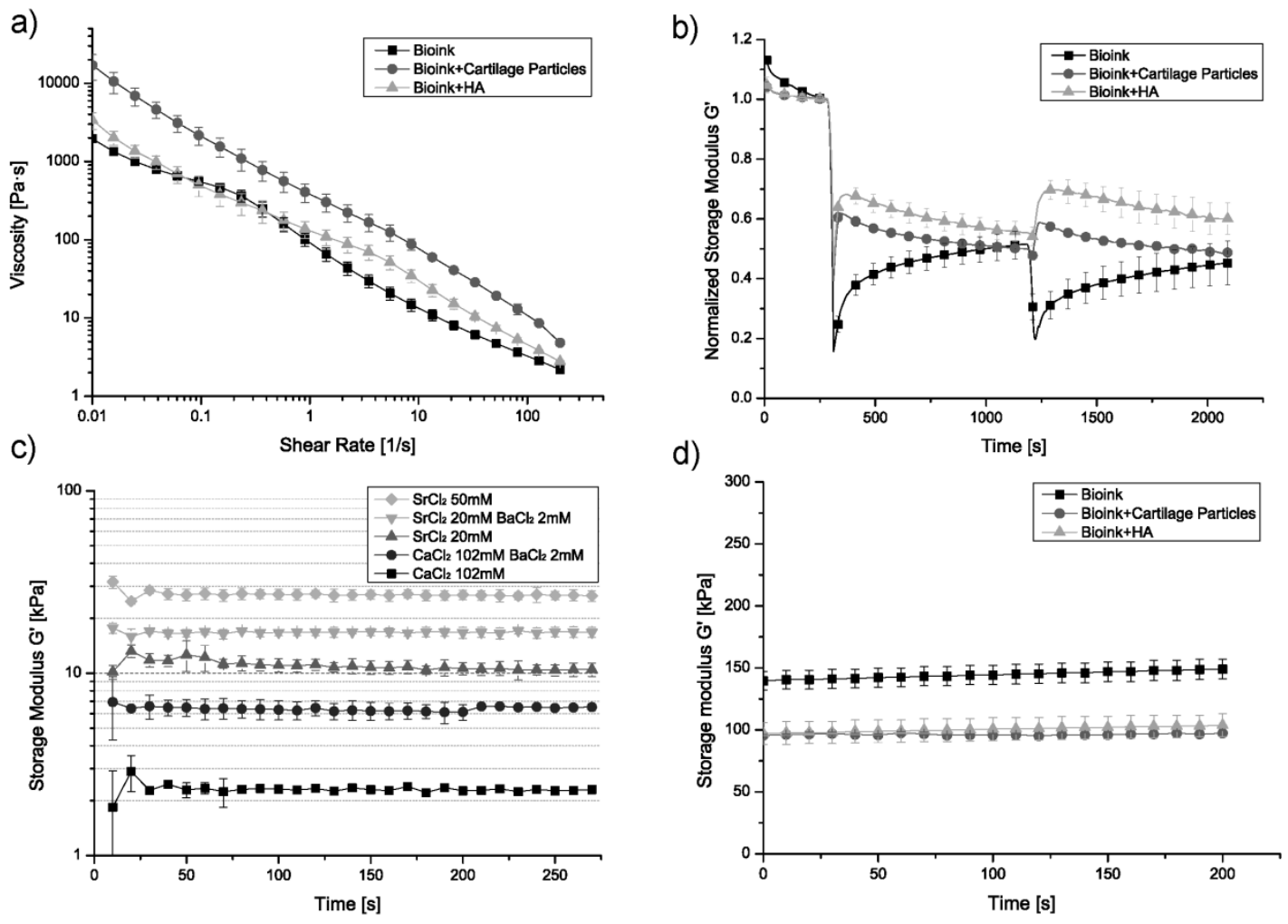

Figure 2. Rheological characterization of the bioink compositions with and without particles. Shear thinning was measured in rotation a), shear recovery in oscillation after shear of 1 second $\left(100^{-s}\right.$ shear rate) for two cycles b), Bioink alone was ionically crosslinked with several cation conditions c), and maximum storage modulus $\mathrm{G}^{\prime}$ of the samples crosslinked for 30 minutes with $20 \mathrm{mM} \mathrm{SrCl}_{2} \mathrm{~d}$ ). Error bars represent standard deviation. 
a)

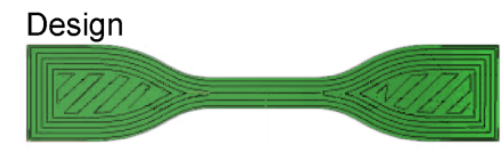

Printed

c)
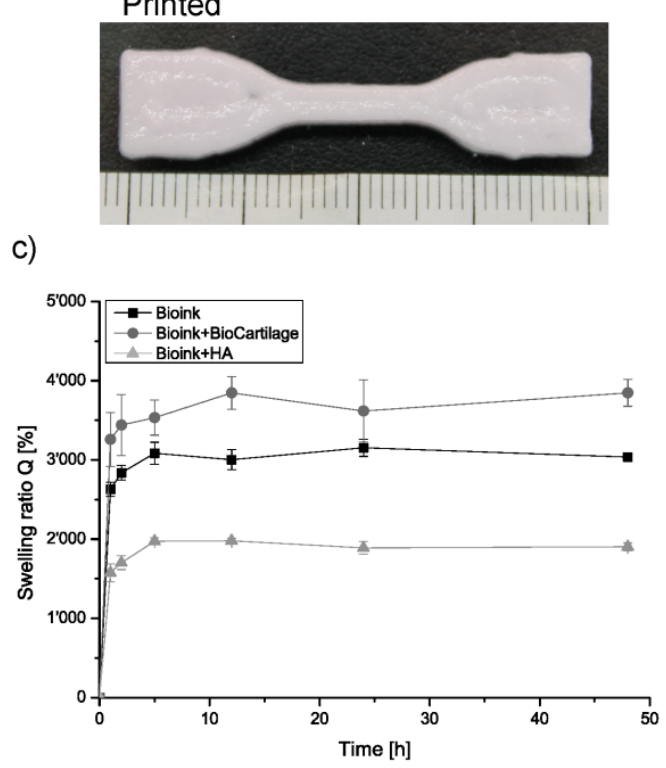
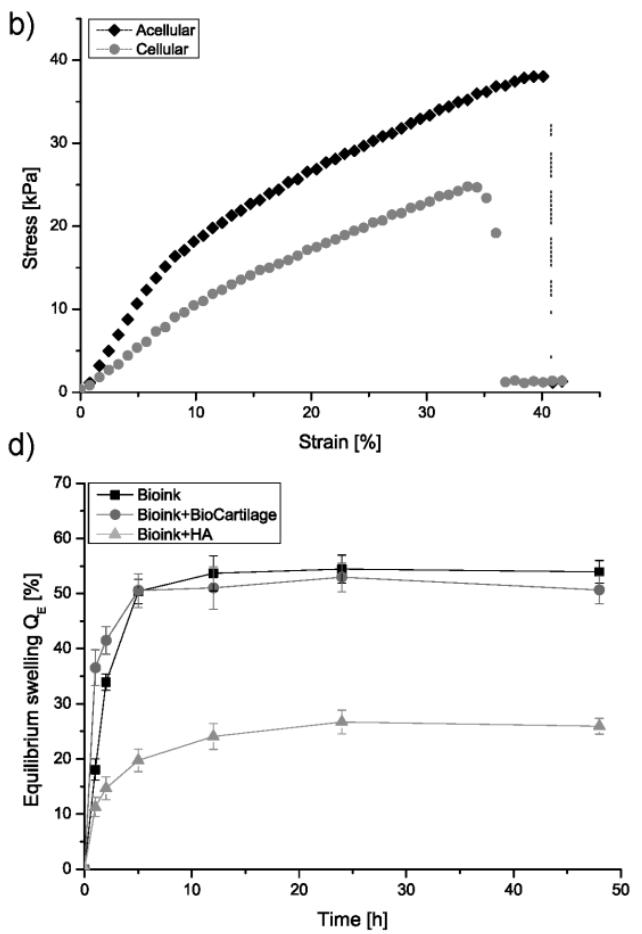

Figure 3. Tensile and swelling properties of the printed constructs. Tensile testing was performed on printed dumbbell specimens where the nozzle path is shown by the black lines and the printed structure is shown after swelling a). Representative stress-strain curves where failure occurred in the central region of the specimen b). Swelling behavior of the bioink compositions based on equation (2) and (3) to evaluate total water retention c) and water retention after crosslinking d) respectively. The smallest divisions on the ruler are $1 \mathrm{~mm}$ and error bars represent standard deviation. 


\section{WILEY-VCH}
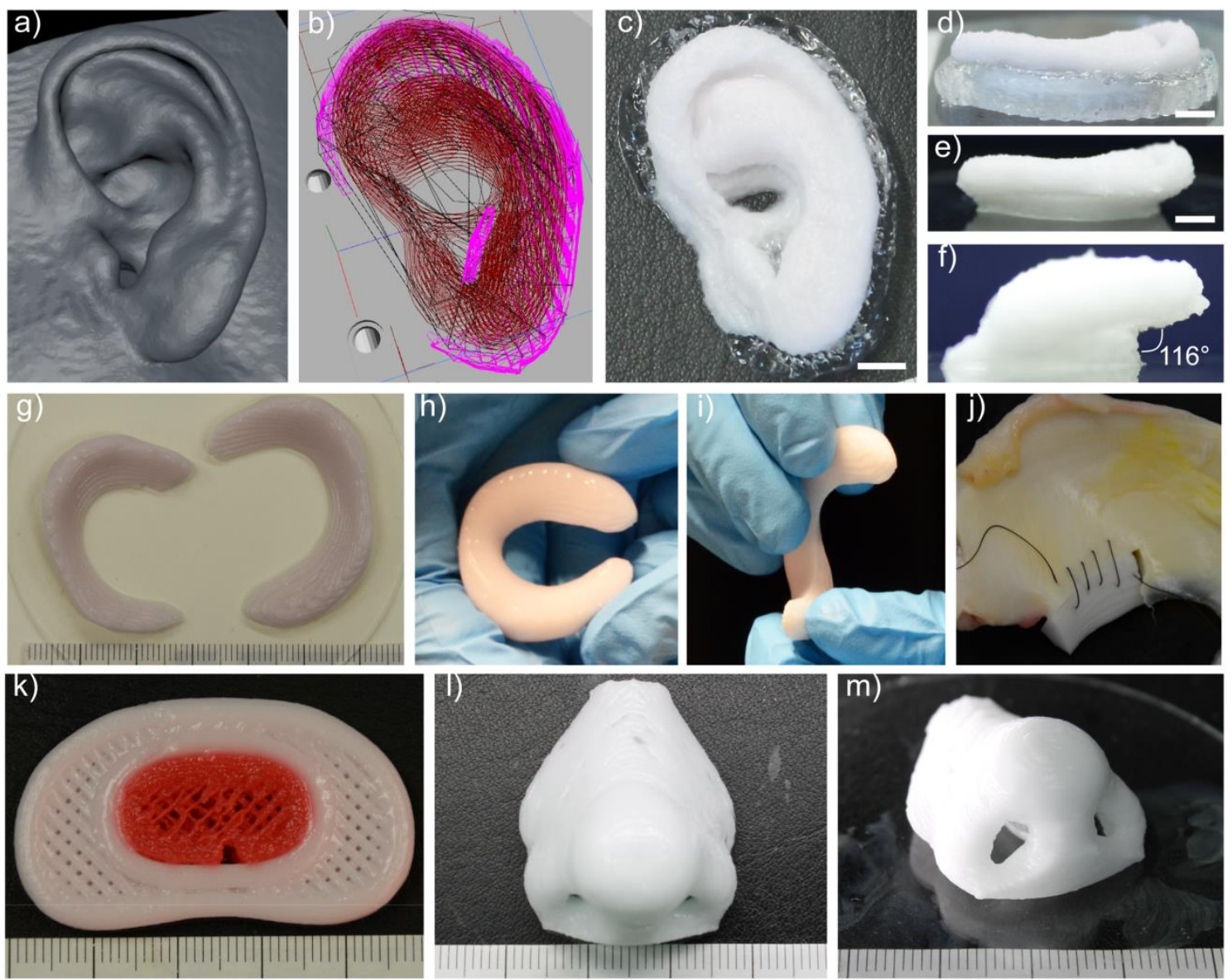

Figure 4. Conversion of image data into three-dimensional cartilage structures. CT scan was thresholded and converted into a .STL file a) which was used to create a printing tool path $($ red $=$ bioink and pink $=$ support $) \mathrm{b}$ ) and external ear graft $\mathrm{c})$. The flexible structure was stable after removing the support and $116^{\circ}$ overhanging structures were observed d-f). Cartilaginous meniscus grafts $\mathrm{g}$ ) were flexible in manipulation $\mathrm{h}-\mathrm{i}$ ) and were stable upon suturing to a bovine meniscus $\mathrm{j}$ ). Cartilaginous grafts such as intervertebral discs $\mathrm{k}$ ) and noses $\mathrm{l}, \mathrm{m}$ ) were printed. Intervertebral disc graft was printed with Bioink+Cartilage particles stained red (nucleus pulposus) and with Bioink $+\mathrm{HA}$ (annulus fibrosus). Scale bars $=5 \mathrm{~mm}$, and the smallest divisions on the ruler are $1 \mathrm{~mm}$. 


\section{WILEY-VCH}

a)

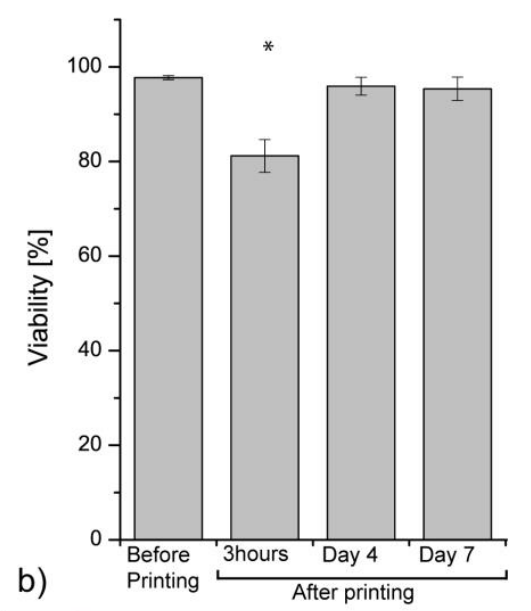

c)
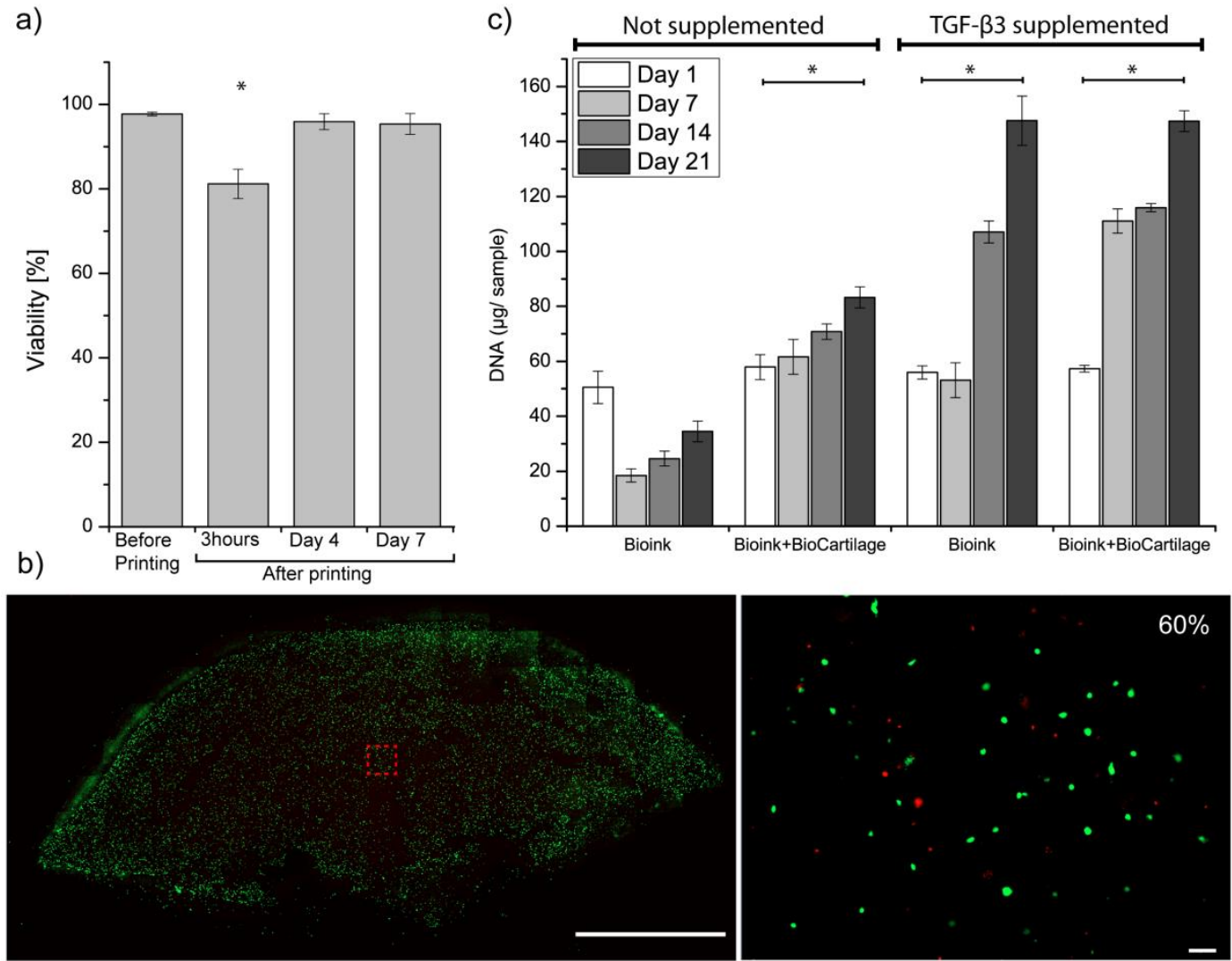

Figure 5. Cell viability of printed constructs and the cell proliferation assay. Viability after printing one layer thick discs was evaluated with live dead staining a) where $80 \%$ viability was observed $3 \mathrm{~h}$ after printing, which recovered to $97 \%$ by day 4 . To assess viability in a large structure, a young adult size nose was printed and the viability was evaluated from a central slice (diffusion distance $\sim 5 \mathrm{~mm}$ ) evaluated by live dead staining. A cell viability of $60 \%$ was observed. Scale bar $5 \mathrm{~mm}$ (left), and $50 \mu \mathrm{m}$ (right). Additionally, cell number in casted disks were evaluated with DNA quantification c) where a statistically significant increase in DNA from day 1 to day 21 was observed with Bioink+BioCartilage and both TGF- $\beta 3$ supplemented compositions. Error bars represent standard deviation and level of significance was $(\mathrm{p}<0.05)$. 


\section{WILEY-VCH}

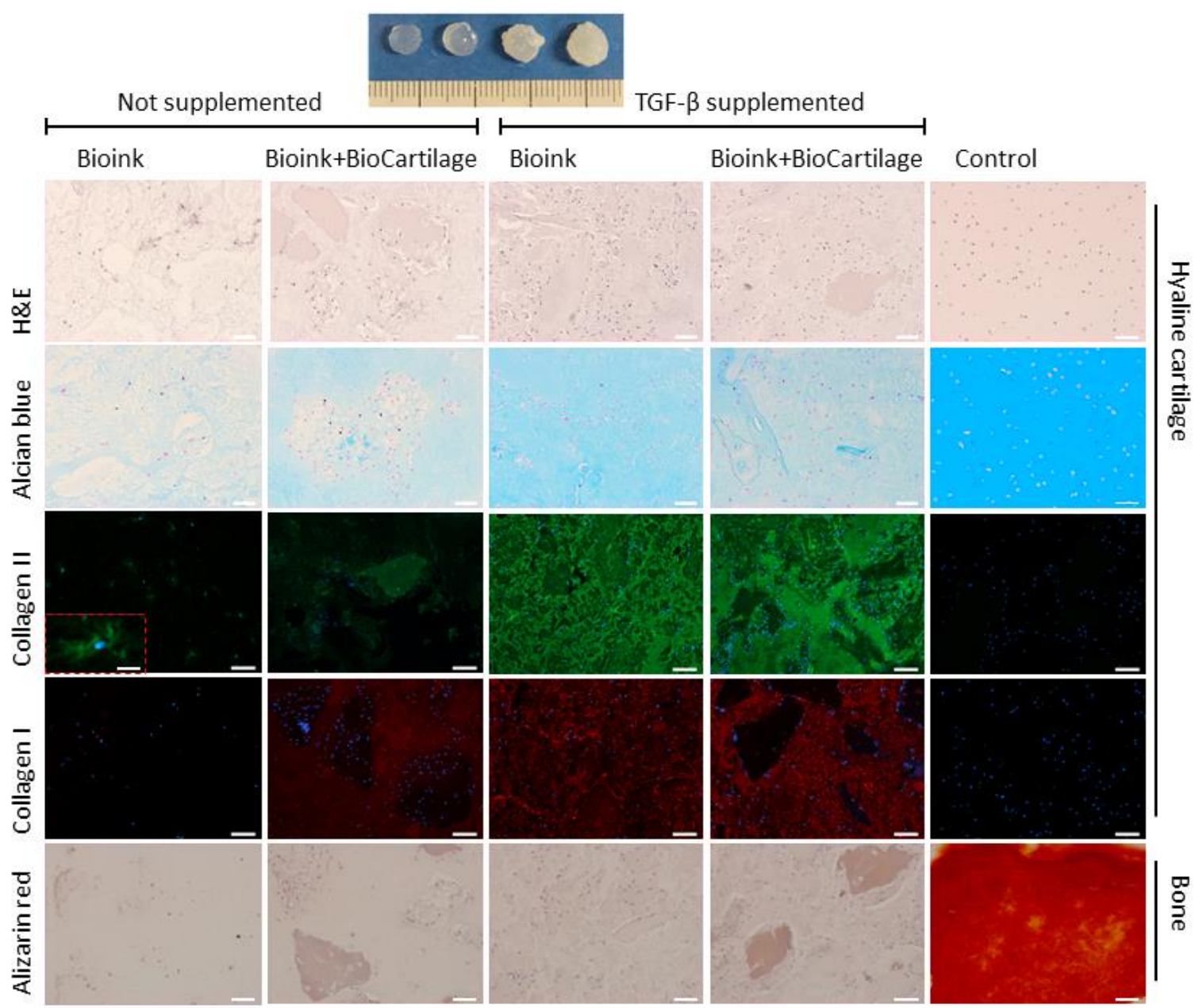

Figure 6. Macroscopic appearance, histology and immunohistochemical stainings after 8 weeks of culture. Bioink alone was not able to stimulate cell proliferation; however good biocompatibility was observed in all the compositionsand collagen II positive pericellular staining was observed (enlargement, scale bar $25 \mu \mathrm{m}$ ). Bioink+BioCartilage enhanced cell proliferation and had a positive effect on GAG production and collagen II depositionas determined by Alcian blue staining. Significant increase in cartilage ECM synthesis was observed in bioink compositions supplemented with TGF- $\beta 3$. No clear difference between Bioink alone and Bioink+BioCartilage was observed. Collagen I was synthesized in the presence of cartilage particles and in TGF- $\beta 3$ supplemented samples. However, no calcification was observed in any condition suggesting a stabile cartilage phenotype. Immunostaining controls were stained without the primary antibody. Scale bars $100 \mu \mathrm{m}$. 

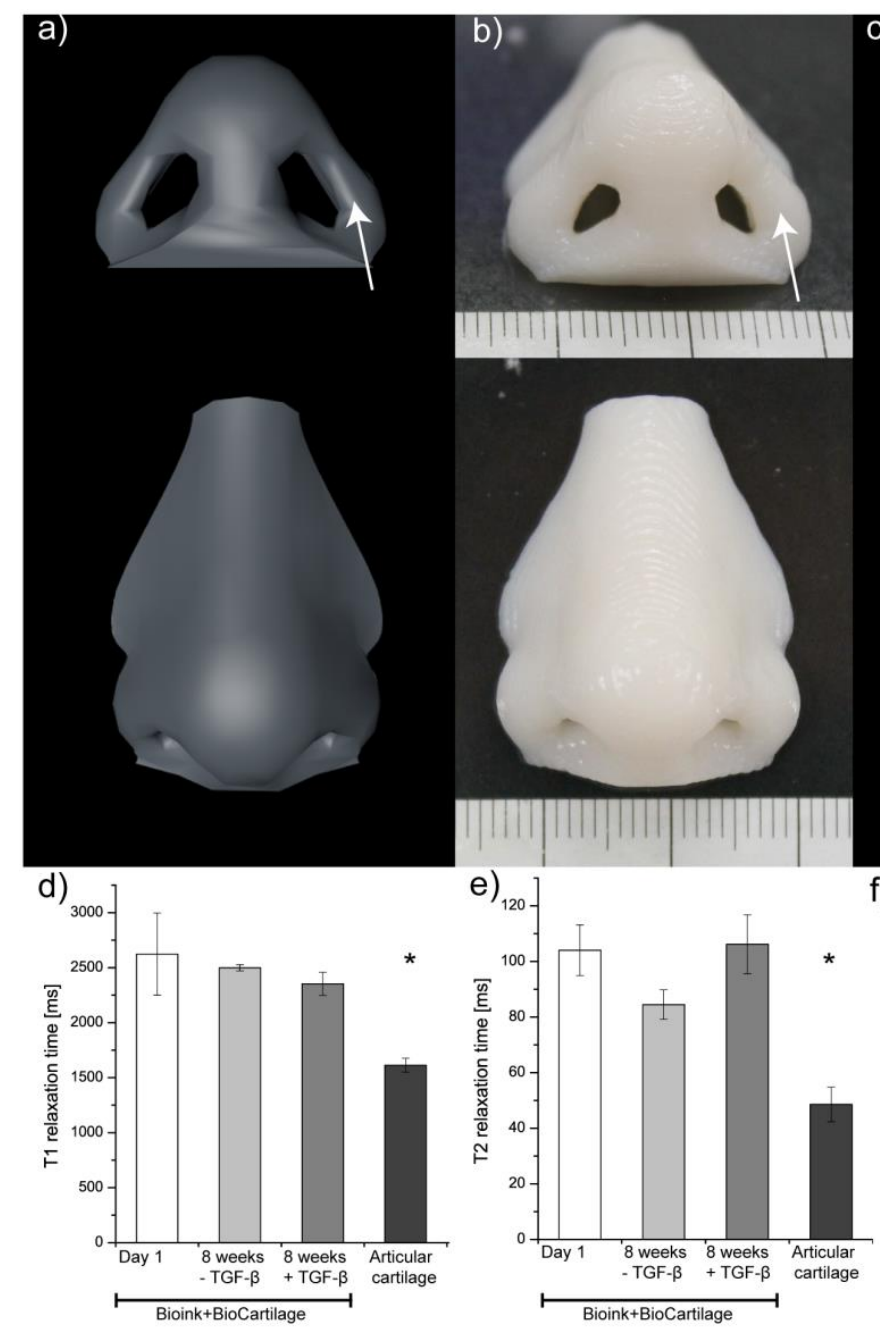

\section{C)}

e)
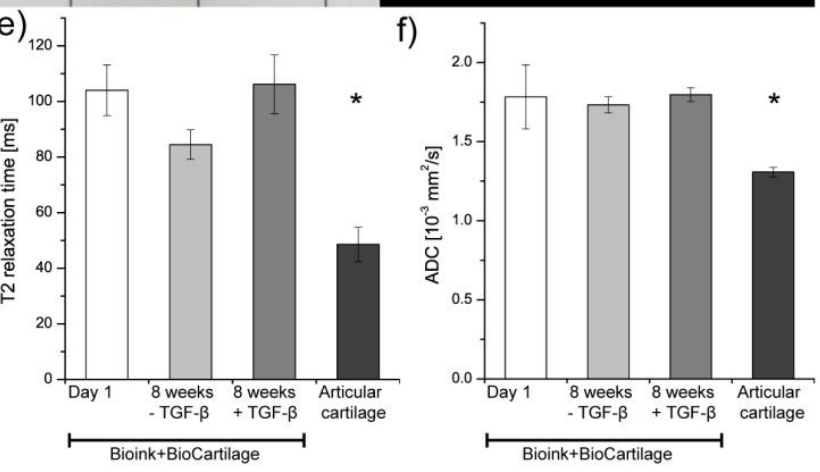

Figure 7. MR imaging for monitoring printed grafts. Nose graft preserved its external dimensions to a good extent after swelling for 2 weeks in PBS. Three-dimensional shape and volume were acquired from the printed nose after two weeks of swelling c) compared to the original 3D model used in the printing a) and the nose immediately after printing $b$ ). The comparison between the original 3D model and the acquired MRI model illustrates slight thickening of the nostril walls (white arrows). Bioink+BioCartilage samples after 8 weeks in vitro with and without TGF- $\beta 3$ were imaged prior histology to evaluate the MRI capability to detect ECM production. Engineered tissue grafts were compared to the initial grafts (day 1) and to bovine hyaline cartilage. T1 d), T2 e) and ADC f) values were measured and_no significant difference between the engineered samples over the culture period was observed. The smallest divisions on the ruler are $1 \mathrm{~mm}$. Error bars represent standard deviation and level of significance was $\mathrm{p}<0.05$. 


\section{WILEY-VCH}

Table 1. Summary of the rheological measurements. The yield points were calculated using the Herschel/Bulkley equation (1).

\begin{tabular}{|l|l|l|l|}
\hline & Bioink & Bioink+HA & Bioink+Cartilage Particles \\
\hline Yield point & $15.6 \mathrm{~Pa} \pm 0.7 \mathrm{~Pa}$ & $17.7 \mathrm{~Pa} \pm 6.5 \mathrm{~Pa}$ & $122 \mathrm{~Pa} \pm 22 \mathrm{~Pa}$ \\
\hline Cessation in $10 \mathrm{~s}^{*}$ & $21 \%$ & $90 \%$ & $98 \%$ \\
\hline Maximum G' & $152 \mathrm{kPa} \pm 3.0 \mathrm{kPa}$ & $110 \mathrm{kPa} \pm 2.0 \mathrm{kPa}$ & $96 \mathrm{kPa} \pm 1.0 \mathrm{kPa}$ \\
\hline
\end{tabular}

* Shear recovery at $10 \mathrm{~s}$ after the second shear sequence. 


\section{WILEY-VCH}

A bioink is presented which allows the printing of complex cell-laden cartilaginous structures with regulatory-compliant biomaterials. Printed grafts are tunable in mechanical properties and tissue-specific, as they can contain extracellular matrix particles. Bioprinting of overhanging structures is achieved using a co-extruded support polymer which also acts as a cation-reservoir to rapidly crosslinking the bioink.

Keywords: Biomedical Applications, Tissue Engineering, Hydrogels

Matti Kesti, Christian Eberhardt, Guglielmo Pagliccia, David Kenkel, Daniel Grande, Andreas Boss, Marcy Zenobi-Wong*

Bioprinting Complex Cartilaginous Structures with Clinically-Compliant Biomaterials
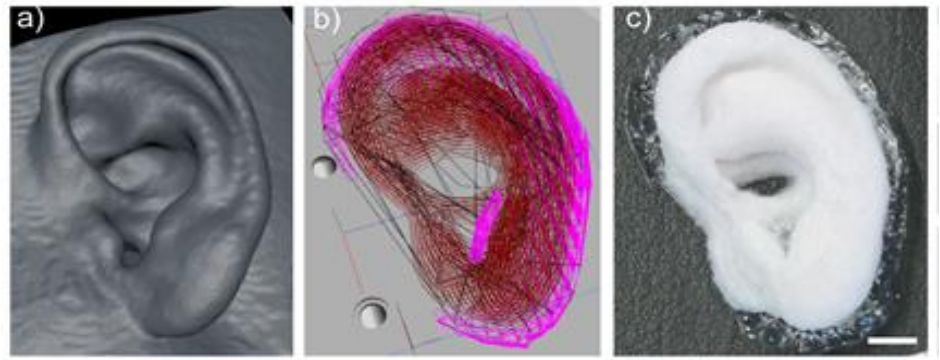


\section{WILEY-VCH}

Copyright WILEY-VCH Verlag GmbH \& Co. KGaA, 69469 Weinheim, Germany, 2013.

\section{Supporting Information}

\section{Bioprinting Complex Cartilaginous Structures with Clinically-Compliant Biomaterials}

Matti Kesti, Christian Eberhardt, Guglielmo Pagliccia, David Kenkel, Daniel Grande, Andreas Boss, Marcy Zenobi-Wong*

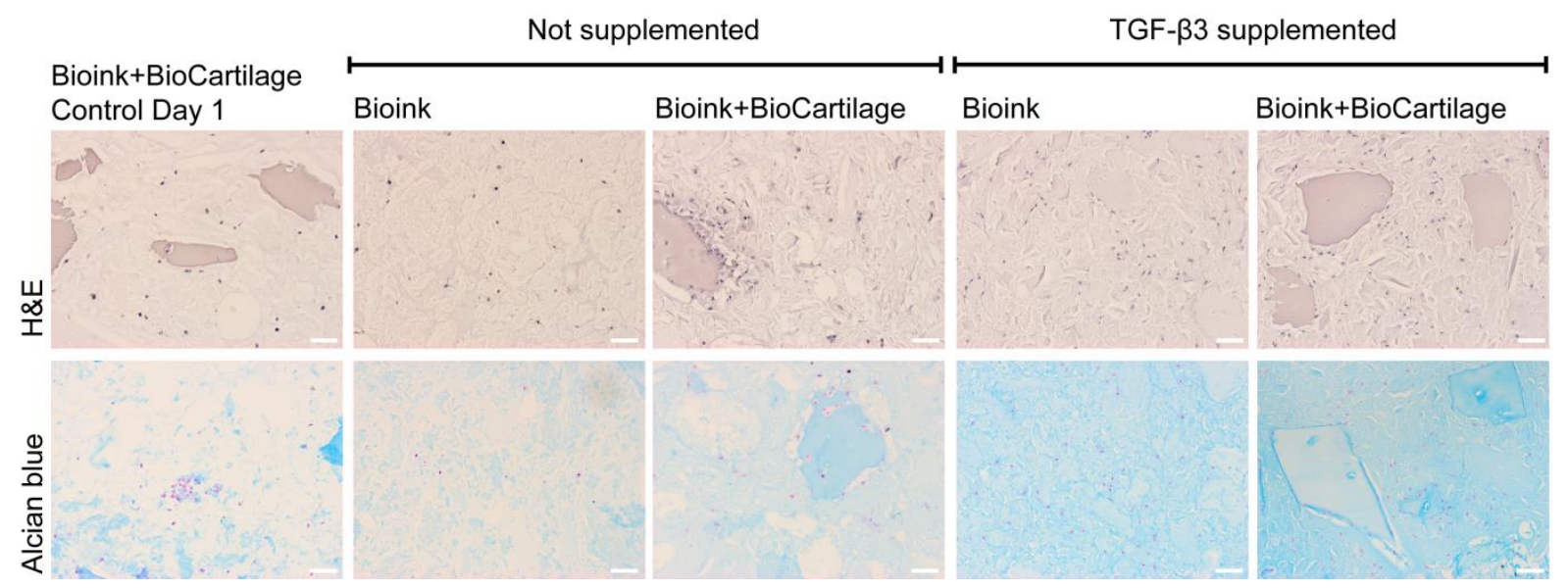

Figure S1. Histology after 3 weeks in culture and the initial scaffold staining. Day 1 Bioink+BioCartilage show light background with $\mathrm{H} \& \mathrm{E}$ and Alcian blue staining due interactions with the biopolymers. Clear increase in cell numbers can be observed in Bioink+BioCartilage and in both TGF- $\beta 3$ supplemented compositions. 
WILEY-VCH

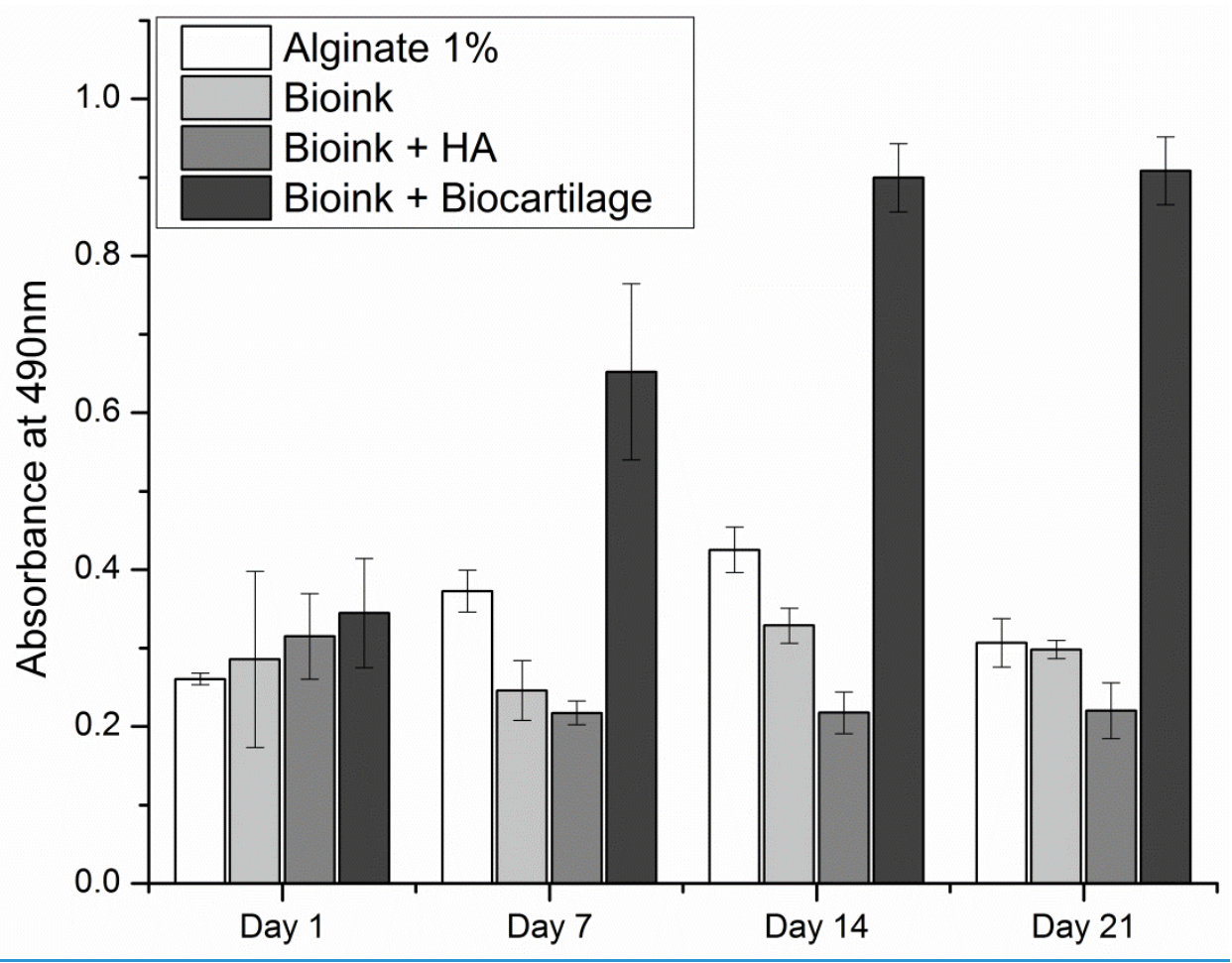

Figure $\mathbf{S} 2$ illustrates the MTS assay over 21 days. Day 1 values were not significantly

different between the groups. Bioink+BioCartilage had significantly higher values at day 7 , $\underline{14}$ and 21 days compared to the alginate controls. 


\section{WILEY-VCH}

Table S1. T1 relaxation values of the cultured scaffolds had a slight decrease compared to day 1 value. Furthermore, T2 relaxation values of the cultured scaffolds were counterintuitive where the Bioink+BioCartilage samples T2 values decreased while TGF- $\beta 3$ supplemented scaffold did not show any decrease despite the clear cartilaginous ECM production observed in histology. Finally, ADC values did not change after 8 weeks. All the differences compared to the native articular cartilage were significant. Data shown as mean \pm standard deviation.

\begin{tabular}{|l|l|l|l|}
\hline Sample and/or time & T1 relaxation & T2 relaxation & ADC \\
\hline Bioink+BioCartilage & $2623 \mathrm{~ms} \pm 374$ & $104 \mathrm{~ms} \pm 9.1$ & $1.78 \times 10^{-3} \mathrm{~mm}^{2} / \mathrm{s} \pm 2.01 \times 10^{-4}$ \\
\hline B weeks & & & \\
\hline Bioink+BioCartilage & $2353 \mathrm{~ms} \pm 105$ & $106 \mathrm{~ms} \pm 11$ & $1.79 \times 10^{-3} \mathrm{~mm}^{2} / \mathrm{s} \pm 4.34 \times 10^{-5}$ \\
8 weeks with TGF- $\beta 3$ & & & \\
\hline Articular cartilage & $1614 \mathrm{~ms} \pm 63$ & $49 \mathrm{~ms} \pm 6$ & $1.3 \times 10^{-3} \mathrm{~mm}^{2} / \mathrm{s} \pm 3.08 \times 10^{-5}$ \\
\hline
\end{tabular}


Click here to download Production Data: Figure 2 Rheology2.tif 
Click here to download Production Data: Figure 3 Mechanical and water retension.tif 
Click here to download Production Data: Figure 5 Cell viability and proliferation.tif 
Click here to download Production Data: Supplementary S1 Histology 3 week.tif 
Click here to download Production Data: Supplementary S2 MTS assay.tif 